\title{
ERROS E EVENTOS ADVERSOS: A INTERFACE COM A CULTURA DE SEGURANÇA DOS PROFISSIONAIS DE SAÚDE
}

\author{
Verusca Soares de Souza ${ }^{1}$, Andressa Morello Kawamoto ${ }^{2}$, João Lucas Campos de Oliveira ${ }^{3}$, Nelsi Salete Tonini ${ }^{4}$, Luciana
} Magnani Fernandes ${ }^{4}$, Anair Lazzari Nicola ${ }^{4}$

1Enfermeira. Doutoranda em Enfermagem. Docente de Enfermagem da Universidade Estadual do Paraná. Paranavaí, PR, Brasil. ${ }^{2}$ Enfermeira. Graduada em Enfermagem pela Universidade Estadual do Oeste do Paraná. Cascavel, PR, Brasil.

${ }^{3}$ Enfermeiro. Doutorando em Enfermagem. Docente de Enfermagem da Universidade Estadual do Oeste do Paraná. Cascavel, PR, Brasil.

${ }^{4}$ Enfermeira. Doutora em Enfermagem. Docente de Enfermagem da Universidade Estadual do Oeste do Paraná. Cascavel, PR, Brasil.

RESUMO: Estudo descritivo com abordagem quantitativa, que objetivou analisar a cultura de segurança em relação aos erros e eventos adversos, na percepção de profissionais de saúde. Foi realizado entre abril e junho de 2014 em três unidades de um hospital de ensino do sul do Brasil. Participaram 71 profissionais de saúde, que responderam ao questionário "Hospital Survey on Patient Safety Culture". A análise ocorreu por meio de estatística descritiva das dimensões que tratam da cultura de segurança em relação à ocorrência de erros e eventos adversos do instrumento. A maioria dos participantes demonstrou percepção de cultura de segurança desfavorável à comunicação sobre as falhas assistenciais e apontou para cultura punitiva ainda presente na instituição, o que pode justificar a quantidade reduzida de notificação de falhas. Conclui-se que há necessidade de disseminação da cultura não punitiva na organização, para que os erros e eventos adversos possam ser notificados, analisados e corrigidos.

DESCRITORES: Segurança do paciente; Cultura organizacional; Erros médicos; Enfermagem.

\section{ERRORS AND ADVERSE EVENTS: THE INTERFACE WITH HEALTH PROFESSIONALS' SAFETY CULTURE}

ABSTRACT:This descriptive study, with a quantitative approach aimed to analyze the safety culture in relation to errors and adverse events in the perception of health professionals. It was undertaken between April and June 2014 in three units of a teaching hospital in the South of Brazil. A total of 71 health professionals participated, responding to the "Hospital Survey on Patient Safety Culture" questionnaire. Analysis took place through descriptive statistics of the dimensions which are to do with the safety culture in relation to the occurrence of errors and adverse events in this instrument. The majority of the participants demonstrated a perception of safety culture which was not favorable to communication regarding care failures and indicated a punitive culture still to be present in the institution, which may explain the underreporting of shortcomings. It is concluded that it is necessary to disseminate the nonpunitive culture in the organization, such that errors and adverse events may be reported, analyzed and corrected.

DESCRIPTORS: Patient safety; Organizational culture; Medical errors; Nursing.

\section{ERRORES Y EVENTOS ADVERSOS: LA INTERFAZ CON LA CULTURA DE SEGURIDAD DE LOS PROFESIONALES DE SALUD}

RESUMO: Estudio descriptivo con abordaje cuantitativo, cuyo objetivo fue analizar la cultura de seguridad acerca de errores y eventos adversos, en la percepción de profesionales de salud. Fue realizado entre abril y junio de 2014 en tres unidades de un hospital de enseñanza del sur de Brasil. Participaron 71 profesionales de salud, que contestaron al cuestionario Hospital Survey on Patient Safety Culture. EI análisis ocurrió por medio de estadística descriptiva de las dimensiones que abordan la cultura de seguridad acerca de la ocurrencia de errores y eventos adversos del instrumento. La mayoría de los participantes demostró percepción de cultura de seguridad desfavorable a la comunicación sobre los errores asitenciales y apuntó para cultura punitiva todavía existente en la institución, lo que puede justificar la cantidad reducida de notificación de errores. Se concluye que hay necesidad de diseminación de la cultura no punitiva en la organización, para que los errores y eventos adversos puedan ser notificados, analizados y corregidos.

DESCRITORES: Seguridad del Paciente; Cultura Organizacional; Errores Médicos; Enfermería. 


\section{INTRODUÇÃO}

Na busca pela qualidade do cuidado à saúde, a garantia da segurança do paciente se constitui um compromisso das instituições e dos profissionais. Desta forma, a redução dos riscos inerentes à prestação da assistência se relaciona diretamente com as mudanças na cultura e nos processos de trabalho adotados pelos serviços de saúde, visto que a assistência produzida e consumida é resultante de um sistema complexo de relações, o que torna passível a ocorrência de erros e/ou eventos adversos no processo do cuidado ${ }^{(1)}$.

Os eventos adversos são definidos como incidentes que resultam em danoà saúde ${ }^{(2)}$. Por sua vez, os erros consistem em falhas na execução de uma ação planejada ${ }^{(3)}$. Embora estejam inseridos em contexto comum, os termos se diferenciam pelo fato de que os eventos adversos remetem-se ao resultado do cuidado, que podem ocorrer em decorrência de erros no processo de prestação da assistência à saúde ${ }^{(3)}$.

Tanto os erros como os eventos adversos podem implicar em aumento do tempo de internação, custos assistenciais e muitas vezes, encargos jurídicos ${ }^{(4)}$. Ao entender a relação da ocorrência dos eventos adversos com os resultados assistenciais, a Aliança Mundial para a Segurança do Paciente foi criada, no intuito de estabelecer metas e protocolos mundiais de atendimento voltados à segurança ${ }^{(3)}$.

Cabe ressaltar que, o estabelecimento de metas e protocolos sem o devido envolvimento do profissional pode resultar em descumprimento das normativas estabelecidas ${ }^{(5)}$. Neste sentido, questiona-se sobre o consumo acrítico dos conhecimentos científicos ${ }^{(5)}$, visto que a implementação parcial de protocolos de atendimento, acrescida da falta de estímulo organizacional, pode comprometer a segurança na assistência, tanto no âmbito dos consumidores (usuários), quanto dos provedores (profissionais e serviços), influindo negativamente na qualidade do cuidado e possivelmente reduzindo a satisfação dos consumidores ${ }^{(6-7)}$.

Mediante o referido entendimento da relação entre a qualidade e segurança do cuidado, o comportamento dos profissionais e o apoio da instituição, iniciou-se o movimento para a promoção da cultura organizacional voltada ao desenvolvimento do atendimento seguro ${ }^{(2)}$. Nesta perspectiva, a análise da cultura de segurança pode ajudar a compreender o funcionamento da instituição e vai além de um programa de qualidade, por envolver elementos relacionados às atitudes e condutas das pessoas ${ }^{(6)}$.

A cultura de segurança pode, então, ser definida como um comportamento individual e organizacional, que busca continuamente estabelecer o compromisso com a busca contínua da minimização dos riscos relacionados à assistência e, consequentemente, auxiliar no alcance da qualidade dos serviços prestados ${ }^{(2)}$. Este bem desejável deve ser comungado por todos os atores presentes nas organizações de saúde dos mais diversos serviços desde a atenção primária $^{(8)}$ até a alta complexidade hospitalar ${ }^{(6)}$.

Ante ao exposto, fica evidente que a postura frente à segurança do paciente fomentada pelas instituições de saúde pode interferir na cultura sistêmica da organização(2), e por isso, considerase que as pesquisas com foco nesta cultura entre os profissionais possam fundamentar ações para o (re)planejamento do cuidado, visando ao atendimento seguro.

Em que pese a importância de entendera cultura de segurança voltada à ocorrência de eventos adversos, estudos que a analisaram entre equipes multidisciplinares indicaram a necessidade de mudanças na abordagem puramente punitiva direcionada aos profissionais ${ }^{(6,9)}$. Isto porque, para que se alcance um cenário de segurança, é necessário que os serviços adotem o comportamento de aprendizagem contínua, no qual a notificação dos eventos adversos e a análise de suas causas sejam elementos disparadores de melhorias nos processos assistenciais, a fim de minimizar a ocorrência de danos evitáveis aos pacientes $^{(2)}$.

Ao destacar a importância e a necessidade de envolvimento dos profissionais de saúde no estabelecimento da cultura de segurança nas instituições de saúde, em especial no que concerne à ocorrência de eventos adversos, questiona-se: Como se apresenta a cultura de segurança em relação aos erros e eventos adversos de profissionais de saúde? Diante desta questão, o objetivo desta pesquisa consiste em analisar a cultura de segurança em relação aos erros e eventos adversos, na percepção de profissionais de saúde.

\section{MÉTODO}

Trata-se de estudo descritivo, transversal, de abordagem quantitativa. O local de pesquisa foram as unidades de internação em Clínica Médica e Cirúrgica Geral; Neurologia Clínica/ 
Cirúrgica e Ortopedia; e Centro Cirúrgico de um hospital de ensino público, localizado em uma região pólo do interior do estado do Paraná, que possui 195 leitos exclusivos do Sistema Único de Saúde (SUS).

A saber, as referidas unidades contam, respectivamente, com 28 e 26 leitos, e cinco salas operatórias. Em geral, recebem pacientes para o cuidado em alta complexidade, visto que o hospital é referência para o atendimento neurológico; ao trauma; tratamento do HIVIAIDS; e emergências clínicas, para uma população de aproximadamente dois milhões de habitantes.

Todos os profissionais de saúde que atuavam diariamente e em todos os turnos de trabalho nas unidades investigadas foram considerados a participar da investigação, quais sejam: enfermeiros, técnicos de enfermagem, auxiliares de enfermagem, médicos, farmacêuticos, fisioterapeutas, e residentes em enfermagem, medicina, farmácia e fisioterapia.

Para os profissionais de enfermagem, os quais eram os únicos que possuíam escala de trabalho fixa, estabeleceu-se o seguinte critério de exclusão: ausência na unidade por férias, licenças, ou outro motivo. Considerou-se recusa à participação, aqueles profissionais que se resignaram em preencher o instrumento de coleta de dados após três tentativas. Como os demais profissionais previstos à participação não possuíam escala de trabalho fixada, se estabeleceu como critério de inclusão dos mesmos estarem presentes nas unidades durante o período de coleta de dados.

Após a aplicação dos critérios de inclusão e exclusão, foram convidados a participar da investigação todos os profissionais (171) atuantes nas unidades, mediante esclarecimento da finalidade do estudo, entrega de questionário e fornecimento do Termo de Consentimento Livre e Esclarecido para leitura e assinatura em duas vias de igual teor, também assinadas pelo pesquisador. A abordagem dos potenciais participantes se deu em local de trabalho durante no início de todos os turnos nas unidades, até o preenchimento da amostra previamente incluída. Ao final dos turnos, o pesquisador retornava aos campos de investigação para recolhimento dos questionários.

Com base nos critérios de elegibilidade e procedimento de abordagem, a amostra constituiu-se de 71 profissionais de saúde que responderamaoquestionário, oquecorrespondeu a $42 \%$ da amostra, valor considerado elevado para pesquisas que incorrem na necessidade do retorno de questionário previamente fornecido ${ }^{(10)}$.

A coleta de dados aconteceu entre abril a junho de 2014, através do questionário autoaplicado intitulado "Hospital Survey on Patient Safety Culture" (HSOPSC), adaptado do instrumento traduzido e validado para o contexto brasileiro ${ }^{(11)}$. Acrescentou-se ao referido instrumento, variáveis que contemplavam a caracterização sociodemográfica e laboral dos participantes.

O HSOPSC contém 42 questões relacionadas à cultura de segurança do paciente, as quais são agrupadas em 12 dimensões ${ }^{(11)}$. Optou-se por analisar as dimensões "Feedback e comunicação a respeito de erros"; "Respostas não punitivas aos erros"; e, "Frequência de relatórios de eventos adversos que são reportados nas diversas modalidades"(11), partindo da prerrogativa de que estas dimensões melhor retratam as percepções dos entrevistados à interface da cultura de segurança com os erros e eventos adversos, e isso, coaduna ao alcance do objetivo previamente estabelecido.

O instrumento utilizado inclui itens que são avaliados a partir de uma escala de Likert de cinco pontos, com categorias de respostas em grau de concordância. A avaliação de cada dimensão e item é estimada com base no percentual de respostas. Valores percentuais mais altos ou mais baixos indicam atitudes positivas/negativas em relação à cultura de segurança do paciente, dependendo da assertiva que foi assinalada no item de avaliação ${ }^{(11)}$. O grau de concordância está disposto às notações " $N$ " - nunca; " $R$ " - raramente; "AV" - às vezes; "QS" - quase sempre; e "S" sempre; e também, "DF" - discordo fortemente; e "D" - discordo; "N" - neutro; "C" - concordo; e "CF" - concordo fortemente.

Os dados coletados à aplicação do formulário de caracterização da amostra e do instrumento foram organizados em planilhas eletrônicas do software Excel, versão 2007. Após isso, o material foi submetido à análise estatística descritiva, com a utilização da mesma ferramenta tecnológica. As variáveis sociodemográficas e referentes ao trabalho foram utilizadas para traçar o perfil da amostra, e as variáveis obtidas na avaliação dos itens do instrumento foram usadas para análise da percepção da cultura de segurança em relação aos erros e eventos adversos.

O projeto desta investigação foi submetido à apreciação do Comitê de Ética em Pesquisa da Universidade Estadual do Oeste do Paraná, e recebeu parecer favorável sob n 558.430/2014. 
Tabela 1 - Características sociodemográficas e laborais dos profissionais de saúde. Cascavel, PR, Brasil, 2014

\begin{tabular}{lc} 
Variáveis & $\mathbf{n}(\%)$ \\
\hline Função & \\
\hline Enfermeiro & $9(12,7)$ \\
\hline Técnico de Enfermagem & $20(28,2)$ \\
\hline Auxiliar de Enfermagem & $12(16,9)$ \\
\hline Médico & $7(9,9)$ \\
\hline Farmacêutico & $1(1,4)$ \\
\hline Fisioterapeuta & $2(2,8)$ \\
\hline Residente de Enfermagem & $5(7)$ \\
\hline Residente de Fisioterapia & $4(5,6)$ \\
\hline Residente de Farmácia & $1(1,4)$ \\
\hline Residente de Medicina & $10(14,1)$ \\
\hline Sexo & \\
\hline Feminino & $47(66,2)$ \\
\hline Masculino & $24(33,8)$ \\
\hline Idade & $17(23,9)$ \\
\hline De 20 a 30 & $23(32,4)$ \\
\hline De 31 a 40 & $14(19,8)$ \\
\hline De 41 a 50 & $1(1,4)$ \\
\hline De 51 a 59 & $16(22,5)$ \\
\hline Não Responderam & $15(21,2)$ \\
\hline Tempo de atuação na instituição & $19(26,7)$ \\
\hline Menos de 1 ano & $12(16,9)$ \\
\hline De 1 a 5 anos & $20(28,2)$ \\
\hline De 6 a 10 anos & $5(7)$ \\
\hline De 11 a 15 anos & \\
\hline Mais de 15 anos & \\
\hline
\end{tabular}

\section{RESULTADOS}

Como mencionado, à luz dos critérios de elegibilidade empregados, houve a participação de 71 profissionais de saúde que atuavam nas unidades investigadas. Destarte, a Tabela 1 apresenta os dados de caracterização da amostra segundo a função, idade, sexo e tempo de atuação na instituição.

Por sua vez, a Tabela 2 sumariza os resultados obtidos às respostas das dimensões da cultura de segurança do paciente "Feedback e comunicação a respeito de erros" e "Frequência de relatórios de eventos que são reportados".

A Tabela 3 apresenta os resultados obtidos à análise da dimensão de cultura de segurança do paciente "Respostas não punitivas aos erros".

A Tabela 4 demonstra a distribuição do número de relatórios de notificação de eventos adversos que foram preenchidos, relatados e entregues pelos profissionais nos últimos 12 meses, de acordo com a função desempenhada.

\section{DISCUSSÃO}

Dentre os 71 profissionais de saúde que participaram do presente estudo, 46 pertenciam à categoria da enfermagem (enfermeiro, técnico de enfermagem, auxiliar de enfermagem ou residente em enfermagem), o que corresponde a $64,8 \%$ do total de sujeitos investigados (Tabela 1 ). A predominância da referida categoria profissional justifica as características sociodemográficas encontradas, visto que $56,3 \%$ de trabalhadores eram adultos jovens e $66,2 \%$ do sexo feminino; o que corrobora com a literatura ${ }^{(9)}$ e com a história da profissão, que associa a figura da mulher à provisão do cuidado.

Quanto ao tempo de atuação, 52,1\% dos profissionais atuam há mais de seis anos na instituição. Tal dado pode ter justificativa na característica do local de estudo, visto tratar-se de um hospital de ensino público e grande parte da contratação da instituição ser realizada por meio de Concurso Público, o que permite ao profissional estabilidade, e consequentemente diminui a rotatividade ${ }^{(12)}$.

A Tabela 2 apresenta a distribuição da frequência das respostas aos itens componentes das dimensões da cultura de segurança, sendo que, a primeira dimensão analisada aborda o Feedback e comunicação a respeito de erros que ocorrem nas unidades. Cabe ressaltar que a falta e/ou falha de comunicação é reconhecida pelos profissionais como um dos fatores que impedem que segurança do paciente seja efetiva ${ }^{(13)}$. Neste aspecto, à luz dos resultados encontrados e da literatura consultada, converge-se à sinalização para a necessidade de promover maior envolvimento dos profissionais responsáveis pela assistência direta ao paciente no planejamento de ações de segurança.

O primeiro item da dimensão Feedback e comunicação a respeito dos erros, questiona acerca do conhecimento dos profissionais em relação às mudanças implantadas em função de eventos adversos notificados. Destaca-se neste item, a maior concentração de respostas negativas ("nunca" e "raramente"); e o fato de que a opção "sempre" não foi assinalada (Tabela 2). Tal dado converge à reflexão de que o retorno aos profissionais quanto às mudanças implantadas ainda não é uma prática rotineira na instituição. 
Tabela 2 - Distribuição das frequências das respostas das dimensões de cultura de segurança do paciente: "Feedback e comunicação a respeito de erros" e "Frequência de relatórios de eventos que são reportados". Cascavel, PR, Brasil, 2014

\begin{tabular}{|c|c|c|c|c|c|c|c|}
\hline Item/Dimensão & & $(\mathrm{N})$ & (R) & $(\mathrm{AV})$ & $(\mathbf{Q S})$ & $(\mathbf{S})$ & TOTAL* \\
\hline \multicolumn{8}{|l|}{ Feedback e comunicação a respeito de erros } \\
\hline \multirow{2}{*}{$\begin{array}{l}\text { Os profissionais têm retorno sobre as } \\
\text { mudanças implantadas em função de eventos } \\
\text { adversos notificados }\end{array}$} & $\mathrm{n}$ & 8 & 26 & 29 & 7 & - & 70 \\
\hline & $\%$ & 11,4 & 37,2 & 41,4 & 10 & & 100 \\
\hline \multirow{2}{*}{$\begin{array}{l}\text { Os profissionais são informados sobre erros } \\
\text { que ocorrem nesta unidade }\end{array}$} & $\mathrm{n}$ & 8 & 22 & 27 & 10 & 3 & 70 \\
\hline & $\%$ & 11,4 & 31,4 & 38,6 & 14,3 & 4,3 & 100 \\
\hline \multirow{2}{*}{$\begin{array}{l}\text { Os profissionais discutem maneiras para } \\
\text { prevenir que erros voltem a ocorrer }\end{array}$} & $\mathrm{n}$ & 8 & 23 & 24 & 12 & 3 & 70 \\
\hline & $\%$ & 11,4 & 32,9 & 34,3 & 17,1 & 4,3 & 100 \\
\hline \multicolumn{8}{|c|}{ Frequência de relatórios de eventos que são reportados } \\
\hline \multirow{2}{*}{$\begin{array}{l}\text { Quando ocorre um erro, ele é percebido e } \\
\text { corrigido antes de afetar o paciente, com que } \\
\text { frequência é notificado? }\end{array}$} & $\mathrm{n}$ & 11 & 24 & 17 & 8 & 10 & 70 \\
\hline & $\%$ & 15,7 & 34,3 & 24,3 & 11,4 & 14,3 & 100 \\
\hline \multirow{2}{*}{$\begin{array}{l}\text { Quando ocorre um erro que não tem potencial } \\
\text { para prejudicar o paciente, com que frequência } \\
\text { é notificado? }\end{array}$} & $\mathrm{n}$ & 11 & 24 & 17 & 9 & 8 & 69 \\
\hline & $\%$ & 16 & 34,8 & 24,6 & 13 & 11,6 & 100 \\
\hline \multirow{2}{*}{$\begin{array}{l}\text { Quando ocorre um erro que poderia prejudicar } \\
\text { o paciente, mas o paciente não é afetado, com } \\
\text { que frequência é notificado? }\end{array}$} & $\mathrm{n}$ & 9 & 26 & 17 & 11 & 6 & 69 \\
\hline & $\%$ & 13 & 37,7 & 24,6 & 16 & 8,7 & 100 \\
\hline
\end{tabular}

Tabela 3 - Distribuição das frequências das respostas da dimensão de cultura de segurança do paciente "Respostas não punitivas aos erros". Cascavel, PR, Brasil, 2014

\begin{tabular}{|c|c|c|c|c|c|c|c|}
\hline Item & & (DF) & (D) & $(\mathrm{N})$ & (C) & (CF) & TOTAL* \\
\hline \multirow{2}{*}{$\begin{array}{l}\text { Os profissionais consideram que seus erros podem } \\
\text { ser usados contra eles }\end{array}$} & $\mathrm{n}$ & 1 & 8 & 10 & 44 & 6 & 69 \\
\hline & $\%$ & 1,4 & 11,6 & 14,5 & 63,8 & 8,7 & 100 \\
\hline \multirow{2}{*}{$\begin{array}{l}\text { Quando ocorre um evento adverso, o profissional } \\
\text { que é apontado e não o problema }\end{array}$} & $\mathrm{n}$ & 1 & 15 & 15 & 35 & 3 & 69 \\
\hline & $\%$ & 1,5 & 21,7 & 21,7 & 50,7 & 4,4 & 100 \\
\hline \multirow{2}{*}{$\begin{array}{l}\text { Os profissionais têm medo que seus erros sejam } \\
\text { registrados nas suas fichas funcionais }\end{array}$} & $\mathrm{n}$ & 1 & 12 & 11 & 43 & 3 & 70 \\
\hline & $\%$ & 1,4 & 17 & 16 & 61,4 & 4,2 & 100 \\
\hline
\end{tabular}

Legenda: DF - discordo fortemente; D - discordo; N - neutro; C - concordo; CF - concordo fortemente.

* Itens em branco não foram considerados

Na mesma dimensão referida anteriormente, quando os profissionais foram questionados se recebem informações acerca dos erros que ocorrem na unidade e se discutem maneiras de prevenir que as falhas voltem a acontecer, apenas três profissionais afirmaram que tal conduta é sempre tomada. Ressalta-se que cabe à gerência investigar e analisar a ocorrência de erros e eventos adversos junto às equipes, pois tal conduta contribui para a implantação de estratégias que reduzem e interceptam as falhas identificadas ${ }^{(3)}$. Para tanto, o fato de os profissionais demonstrarem que os entraves que existem no processo de trabalho não são discutidos, corrobora a percepção de cultura desfavorável à segurança do paciente.
Compreender que os mecanismos que levam à ocorrência da falha, por meio da análise dos processos que desencadearam o erro, permitem que ações preventivas sejam planejadas e que um ambiente educativo seja estabelecido(6). Entretanto, neste estudo, profissionais afirmam não notificar a ocorrência de eventos adversos, quando tal evento não afetou diretamente o paciente (Tabela 2).

Para que seja possível prover um ambiente colaborativo em prol da segurança ao paciente, o estabelecimento de linhas de comunicações efetivas e o apoio dos gestores pode se apresentar como estratégia. Isso porque linhas de comunicação verticalizadas e formais, relacionamse às práticas de gestão pautadas na administração 
Tabela 4 - Notificações de eventos adversos, por função desempenhada na instituição, nos últimos 12 meses. Cascavel, PR, Brasil, 2014

\begin{tabular}{|c|c|c|c|c|c|c|c|}
\hline Categoria & & Nenhum & De 1 a 5 & $\begin{array}{c}\text { De } 6 \text { a } \\
10\end{array}$ & $\begin{array}{c}\text { De } 11 \text { a } \\
15\end{array}$ & $\begin{array}{c}\text { Mais de } \\
20\end{array}$ & TOTAL \\
\hline \multirow[t]{2}{*}{ Enfermeiro } & $\mathrm{n}$ & 3 & 5 & - & 1 & - & 9 \\
\hline & $\%$ & 33,3 & 55,6 & & 11,1 & & 100 \\
\hline \multirow[t]{2}{*}{ Técnico de Enfermagem } & $\mathrm{n}$ & 16 & 4 & - & - & - & 20 \\
\hline & $\%$ & 80 & 20 & & & & 100 \\
\hline \multirow[t]{2}{*}{ Auxiliar de Enfermagem } & $\mathrm{n}$ & 8 & 4 & - & - & - & 12 \\
\hline & $\%$ & 66,7 & 33,3 & & & & 100 \\
\hline \multirow{2}{*}{$\begin{array}{l}\text { Médico do Corpo } \\
\text { Clínico }\end{array}$} & $\mathrm{n}$ & 5 & 2 & - & - & - & 7 \\
\hline & $\%$ & 71,4 & 28,6 & & & & 100 \\
\hline \multirow[t]{2}{*}{ Farmacêutico } & $\mathrm{n}$ & - & 1 & - & - & - & 1 \\
\hline & $\%$ & & 100 & & & & 100 \\
\hline \multirow[t]{2}{*}{ Fisioterapeuta } & $\mathrm{n}$ & 1 & - & 1 & - & - & 2 \\
\hline & $\%$ & 50 & & 50 & & & 100 \\
\hline \multirow[t]{2}{*}{ Residente de Medicina } & $\mathrm{n}$ & 9 & 1 & - & - & - & 10 \\
\hline & $\%$ & 90 & 10 & & & & 100 \\
\hline \multirow[t]{2}{*}{ Residente de Enfermagem } & $\mathrm{n}$ & 3 & 2 & - & - & - & 5 \\
\hline & $\%$ & 60 & 40 & & & & 100 \\
\hline \multirow[t]{2}{*}{ Residente de Fisioterapia } & $\mathrm{n}$ & 4 & - & - & - & - & 4 \\
\hline & $\%$ & 100 & & & & & 100 \\
\hline \multirow[t]{2}{*}{ Residente de Farmácia } & $\mathrm{n}$ & 1 & - & - & - & - & 1 \\
\hline & $\%$ & 100 & & & & & 100 \\
\hline
\end{tabular}

clássica e autocrática e; no contexto dos serviços de saúde, podem proporcionar distância entre os profissionais responsáveis pelo cuidado direto e seus gestores, evidenciando a distribuição desigual do poder pela formalização exacerbada do organograma e, consequentemente, gerando relações de trabalho rígidas e impessoais ${ }^{(14)}$.

A falta de linhas de comunicação efetivas é reconhecida com uma dentre várias situações que favorecem o desencadeamento de erros no cotidiano de trabalho e diminui a adesão à notificação de eventos adversos por parte dos profissionais, devido ao receio de possível punição(6), o que pode impedir o fomento à cultura de segurança nesse aspecto. Em consonância à afirmativa anterior, $72,5 \%$ dos profissionais consideram que seus erros podem ser usados contra eles; e $65,6 \%$ afirmaram ter medo que seus erros sejam registrados em suas fichas funcionais (Tabela 3). Estes achados ratificam estudo que teve por objetivo verificar a frequência de eventos adversos e a existência de punição segundo a percepção dos enfermeiros em terapia intensiva, o qual afirmou que a cultura punitiva ainda persiste no setor ${ }^{(15)}$.

A procura pelos responsáveis pelos erros, ao invés da análise dos processos que culminaram no erro, pode ser considerada como uma conduta contraproducente e ineficiente para almejar o atendimento seguro. Esse pensamento é corroborado por pesquisa que objetivou avaliar a qualidade do processo de atendimento de um serviço de urgência público e apontou que a excelência não é obtida pela identificação e punição dos culpados, mas sim pela prevenção e/ou análise dos erros/inconformidades, favorecendo as melhorias nos processos de cuidado $^{(16)}$.

A análise crítica da realidade e as ações educativas são condutas reconhecidas pelos enfermeiros responsáveis por programas de gerenciamento de risco como melhores práticas para a segurança do paciente ${ }^{(17)}$. Desta forma, utilizar a ocorrência de eventos como uma oportunidade de orientação dos profissionais quanto às implicações éticas, legais e sociais da ocorrência de falhas, pode sensibilizar as equipes de saúde acerca da importância de sua notificação; contudo, os profissionais entrevistados demonstram que a notificação de erros e eventos adversos não é uma prática comum na realidade investigada. 
Reforça a assertiva anterior a Tabela 4, que apresenta o número de notificações de eventos adversos por categoria profissional e evidencia a possível quantidade reduzida de comunicação de falhas assistenciais, em especial entre os residentes de fisioterapia e farmácia, que afirmaram não ter notificado nenhum evento adverso nos últimos 12 meses. Isso é relevante, pois, sabidamente, a residência é ainda uma etapa da formação dos profissionais e por isso pode servir como subsídio à incorporação da cultura favorável à segurança, incluindo a notificação de falhas.

Um baixo número de notificações de eventos adversos pode estar relacionado ao sistema adotado pela instituição, que é informatizado e incorre na necessidade de o profissional identificar-se ao reportar o erro/evento adverso. Destarte, pesquisa semelhante realizada em um hospital de grande porte da região sul do Brasil, investigou as notificações de eventos adversos. Foi possível constatar que um sistema de notificação em que o profissional que notifica o incidente precisa ser identificado, pode levar à subnotificação ${ }^{(18)}$.

Acredita-se que o baixo número de notificações de eventos adversos pode ter relação com a percepção dos profissionais quanto às respostas punitivas às suas falhas (Tabela 3). Pesquisas que objetivaram mensurar o clima de segurança entre equipes multidisciplinares, indicam que há necessidade de mudança na abordagem puramente punitiva direcionada aos profissionais, pois é preciso compreender a complexidade assistencial que permeia os sistemas de saúde, para então corrigir as falhas nos processos ${ }^{(1,6)}$.

A equipe de enfermagem apresentou o maior número de notificações em relação às outras categorias (Tabela 4). Tal dado pode ter justificativa atrelada aos fatos de que a categoria representa o maior contingente do capital humano nas instituições hospitalares; permeia todos os processos assistenciais desenvolvidos; e no reconhecimento dessa equipe no seu papel importante na assistência, embora enfrente grande resistência baseada no modelo de saúde marcado pela hegemonia médica ${ }^{(19)}$.

Possivelmente, cabe às lideranças adotarem posturas receptivas e estabelecerem linhas de comunicação efetivas com aqueles que prestam o cuidado direto, e assim, a equipe possa buscar em conjunto estratégias de notificação e análise dos erros e eventos adversos. Isto porque a análise da ocorrência destes eventos indesejáveis orienta novas práticas e desta forma, evita a repetição das falhas $^{(19)}$.

A investigação dos eventos adversos permite também que ações educativas sejam estabelecidas tanto na educação em serviço, quanto nos cursos técnicos, de graduação e pósgraduação. Destaca-se que a Política Nacional de Segurança do Paciente (PNSP) prevê a inclusão de conteúdos de segurança no processo de formação profissional $^{(2)}$, o que reforça a necessidade de ampliar o escopo do ensino sobre segurança desde a formação profissional ${ }^{(20)}$. Neste aspecto, levanta-se a hipótese de que profissionais formados (na educação formal e em serviço) em consonância aos preceitos da segurança do paciente poderão aderir à cultura em prol deste bem de forma efetiva.

\section{CONCLUSÃO}

Através desta pesquisa, foi possível identificar que os profissionais recebem com pouca frequência informações acerca da ocorrência de erros e eventos adversos e sobre as mudanças implantadas em decorrência de falhas assistenciais nas unidades pesquisadas. Ainda, existe quantidade reduzida de notificação de erros e eventos adversos, bem como a maior parcela dos profissionais sinaliza que a instituição ainda tem uma postura punitiva às ocorrências.

Conclui-se que há necessidade de maior respaldo aos profissionais em relação aos erros e eventos adversos por parte de seus líderes, já que a cultura de segurança pode ser um reflexo da gestão do serviço. Além disso, o apoio da instituição pode significar estímulo para que os erros possam ser notificados, analisados e corrigidos, impedindo assim a sua repetição.

Como principal limitação deste estudo, citase a ausência de análise estatística inferencial. Em contraponto, acredita-se que esta pesquisa contribui ao avanço na área da saúde por apresentar uma perspectiva importante da cultura de segurança no ambiente hospitalar, que é a sua interface com os erros e eventos adversos; e isso, pode subsidiar tomadas de decisão que favoreçam medidas em prol da segurança do atendimento hospitalar neste aspecto.

Por fim, sugere-se que novas pesquisas sejam fomentadas, com diferentes abordagens metodológicas e/ou objetos de pesquisa, ao exemplo de estudos qualitativos que visem desvelar os diversos tabus que permeiam os erros e eventos adversos. 


\section{AGRADECIMENTOS}

Ao Centro de Ciências Biológicas e da Saúde da Universidade Estadual do Oeste do Paraná, pelo apoio ao desenvolvimento desta pesquisa.

\section{REFERÊNCIAS}

1. Nascimento NB, Travassos CMR. Medical errors and violation of rules and standards in health: a theoretical discussion in the area of patient safety. Physis. 2010; 20(2):625-51.

2. Ministério da Saúde (BR). Portaria n. 529, de 01 de abril de 2013. Institui o programa nacional de segurança do paciente. Diário Oficial da União, Brasília, 02 abr. 2013.

3. Ministério da Saúde (BR). Agência Nacional de Vigilância Sanitária. Documento de referência para o Programa Nacional de Segurança do Paciente. Brasília, 2014.

4. Rutberg $H$, Risberg MB, Sjödahl R, Nordqvist $P$, Valter L, Nilsson L. Characterisations of adverse events detected in a university hospital: a 4-year study using the Global Trigger Tool method. BMJ Open. 2014; 4(5). Disponível: http://bmjopen.bmj.com/content/4/5/ e004879

5. Malik AM. Qualidade e avaliação nos serviços de saúde: uma introdução. In: Feldeman LB, D' Innocenzo M, organizadores. Indicadores, auditorias e certificações: ferramentas de qualidade para gestão em saúde. São Paulo: Martinari; 2010. (p. 21-36)

6. Mello JF, Barbosa SF. Patient safety culture in intensive care: nursing contributions. Texto contexto - enferm. 2013; 22(4):1124-33.

7. Freitas JS, Silva AEBC, Minamisava R, Bezerra ALQ, Sousa MRG. Quality of nursing care and satisfaction of patients attended at a teaching hospital. Rev. LatinoAm. Enfermagem. 2014; 22(3):454-460.

8. Paese F, Sasso GTMD. Patient safety culture in primary health care. Texto contexto - enferm. 2013; 22(2):302-310.

9. Rigobello MCG, Carvalho REFL, Cassiani SHB, Galon T, Capucho HC, Deus NN. The climate of patient safety: perception of nursing professionals. Acta Paul. Enferm. 2012; 25(5):728-735.

10. Thomas JR, Nelson JK, Silverman SJ. Métodos de pesquisa em atividade física. Porto Alegre: Artmed; 2012.

11. Clinco SDO. O hospital é seguro? Percepções de profissionais de saúde sobre segurança do paciente [dissertação]. São Paulo (SP): Escola de Administração de Empresas - Faculdade Getúlio Vargas; 2007.
12. Stacanto K, Zilli PT. Fatores geradores de rotatividade dos profissionais de saúde: uma revisão de literatura. Rev adm saúde. 2010; 12(47):87-99.

13. Ques AAM, Montoro CH, González MG. Strenghts and threats regarding the patients safety: nursing professionals opinion. Rev. Latino-Am. Enfermagem. 2010; 18(3):42-49.

14. Montezeli JH, Peres AM, Bernardino E. Desafios para a mobilização de competências gerenciais por enfermeiros em pronto socorro. Cienc Cuid Saúde. 2014;13(1): 137-144.

15. Claro CM, Krocockz DVC, Toffolleto MC, Padilha KG. Adverse events at the intensive care units: nurses' perception about the culture of no-punishment. Rev. Esc. Enferm. USP, 2011; 45(1):162-7.

16. Silva LG, Matsuda LM. Um olhar para a qualidade no processo de atendimento em um serviço de urgência público. Cienc Cuid Saúde. 2012; 11(suppl):121-128.

17. Costa VT, Meirelles BHS, Erdmann AL. Best practice of nurse managers in risk management. Rev. LatinoAm. Enfermag. 2013; 21(5):1165-1171.

18. Lorenzini E, Santi JAR, Báo ACP. Patient safety: analysis of the incidents notified in a hospital, in south of Brazil. Rev. Gaúcha Enferm. 2014; 35(2):121-127.

19. Magalhães ANM, Riboldi CO, Dall'Agnoll CM. Planning human resources in nursing: challenge for the leadership. Rev Bras Enferm. [Internet] 2009; 62(4) [acesso em 10 jul 2015]. Disponível: http:// www.scielo.br/scielo.php?script=sci_arttext\&pid =S0034-7167200900040002020.

20. Bogarin DF, Zanetti ACB, Machado JP, Gabriel CS, Bernardes A. Segurança do paciente: conhecimento de alunos de graduação em enfermagem. Cogitare enferm. 2014; 19(3):491-7. 